Path. Microbiol. 1970;35:36

\title{
Prognosis and Natural History in the Cardiomyopathies
}

C.M.

\author{
Oakley
}

\section{Department of Clinical Cardiology, Royal Postgraduate Medical School, London}

Abstract
Almost 200 patients with cardiomyopathy have been studied at the Royal Postgraduate Medical School London over
the last 8 years. Roughly half fell into the hypertrophic class without left ventricular dilation. Epidemiological survey
has shown a familial incidence in about one third of these and a dominant mode of inheritance. Inflow restriction and
sudden death predominated in this group. The sporadic cases more often showed left ventricular outflow tract
obstruction and a primary role for mitral regurgitation in the genesis of this outflow obstruction has been postulated. In
the sporadic group a translation from outflow to inflow obstruction sometimes occurs but seems to represent a late
phase of the disorder in contrast to the familial cases in which this may be an early or sole feature. In the congestive
cardiomyopathies with left ventricular dilatation a correlation between cavity dilatation and wall thickness aided
assessment of prognosis the outlook being worse in those with high cavity wall thickness ratios. Coronary
angiography estimations of myocardial blood flow and myocardial biopsy has been carried out in some of the patients
from both groups of cardiomyopathies and the further information gained from these studies was discussed in
conjunction with the physiological hemodynamic and angiographic findings in the whole series of patients with
cardiomyopathy.

